\title{
Direct calibration chain for hand torque screwdrivers from the national torque standard
}

\author{
Koji Ogushi, Atsuhiro Nishino, Koji Maeda and Kazunaga Ueda \\ National Metrology Institute of Japan, AIST, Tsukuba Central 3, 1-1-1 Umezono, Tsukuba, Ibaraki, 305-8563, Japan
}

\section{ABSTRACT}

Hand torque screwdrivers are used for the fastening control of screws in almost all precise mechanical and electrical parts. This paper describes the realization of a direct calibration chain for hand torque screwdrivers traceable to the national torque standard. The calibration methods for a reference torque screwdriver using a primary torque standard machine, a torque screwdriver tester using the reference torque screwdriver, and hand torque screwdrivers using the torque screwdriver tester are described respectively. Uncertainty evaluation methods for each calibration level are also explained. The effectiveness of the calibration chain for hand torque screwdrivers could be demonstrated.

\section{Section: RESEARCH PAPER}

Keywords: Torque; Standard; Screwdriver; Calibration

Citation: Koji Ogushi, Atsuhiro Nishino, Koji Maeda and Kazunaga Ueda, Direct calibration chain for hand torque screwdrivers from the national torque standard, Acta IMEKO, vol. 4, no. 2, article 6, June 2015, identifier: IMEKO-ACTA-04 (2015)-02-06

Editor: Paolo Carbone, University of Perugia, Italy

Received October 10, 2014; In final form January 30, 2015; Published June 2015

Copyright: @ 2015 IMEKO. This is an open-access article distributed under the terms of the Creative Commons Attribution 3.0 License, which permits unrestricted use, distribution, and reproduction in any medium, provided the original author and source are credited

Corresponding author: Koji Ogushi, e-mail: kji.ogushi@aist.go.jp

\section{INTRODUCTION}

Hand torque screwdrivers (HTDs) are necessary for the assembly of medical, electrical, and precision devices in the production control process. There has been some progress in the development of calibration chains for hand torque wrenches (HTWs) in the last two decades [1]-[4]. However, the developmental progress of calibration chains for HTDs has been less reported.

The authors have presented a comparison of the results for torque screwdriver testers (TDTs) by using a reference torque screwdriver (RTD) and a conventional weights-and-bar-system (WBS) and have shown some of the advantages of a calibration chain including an RTD [5]. Parasitic transverse forces and bending moments necessarily occur superposing on pure torque during calibration using WBS. Figure 1 shows the calibration chain for HTDs with uncertainties (approximate) of calibrations and maximum permissible errors of tests for devices which are subject to. The National Metrology Institute of Japan (NMIJ) has proposed this direct torque traceability system for hand torque screwdrivers, cooperating with Japanese industry. First, a HTD should be tested by using torque screwdriver checker (TDC; torque screwdriver testing equipment without loading system) or TDT (torque screwdriver testing equipment with loading system) with the maximum permissible deviation of four percent or six percent according to ISO 6789 [6], which is a standard documentation for requirements and test methods of hand torque tools. Second, the TDC or the TDT should be calibrated by using the RTD within the uncertainty of one percent. Next, the RTD should be calibrated in an JCSS Japan Calibration Service System) accredited laboratory within the uncertainty of approximately $0.2 \%$ to $0.5 \%$. The accredited laboratory has a torque calibration machine (TCM) for torque measuring devices (pure torque loading). The TCM should have a calibration and measurement capability of approximately $0.04 \%$ to $0.2 \%$. A high-precision TMD is used for calibration and control of TCM in the accredited laboratory. Finally, this high-precision TMD should be calibrated by NMIJ within uncertainty of approximately $0.01 \%$ to $0.04 \%$.

This paper describes the establishment of a complete calibration chain from the national torque standard to HTDs, where the calibration and testing methods for the RTD using a national torque standard machine (TSM), for the TDT using the RTD, and for HTDs using the TDT are also investigated. It is shown that the traceability of the test results for HTDs is experimentally confirmed by this chain.

The torque range used for HTDs is generally small, e.g., from several centi-Newton meters to several Newton meters. National torque standards at such a small range had not been 


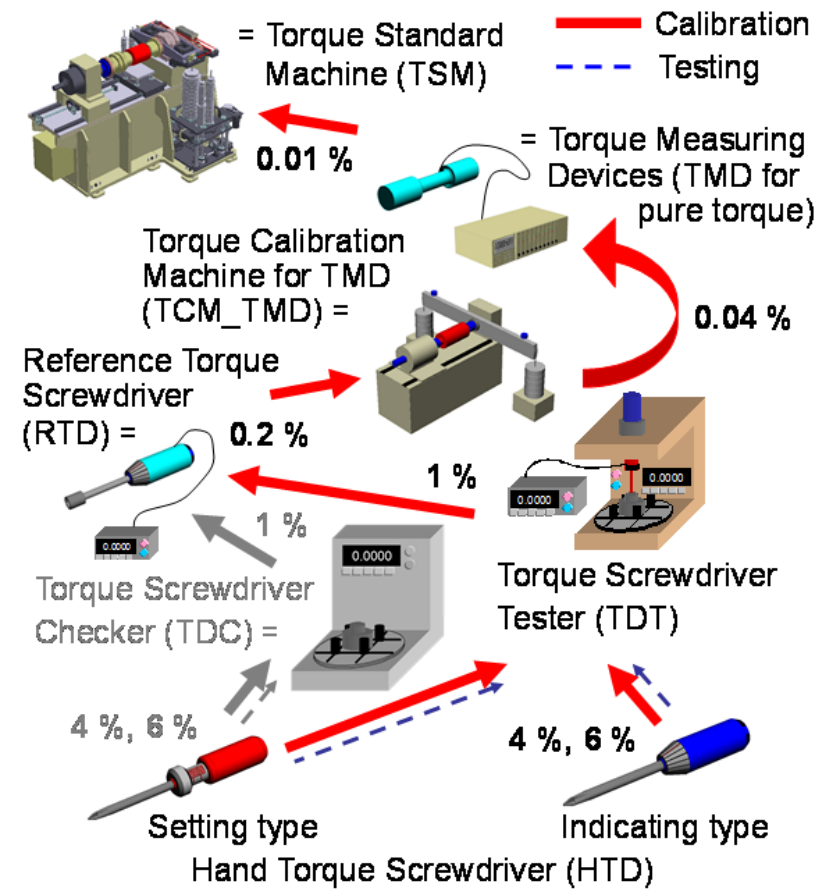

Figure 1. Torque traceability system for HTDs proposed by NMIJ (percentages show the maximum permissible errors or approximate demanded uncertainty).

established in many countries, so the development of a calibration chain for HTDs has not progressed so much until recently. At NMIJ, a new deadweight torque standard machine $(10-\mathrm{N} \cdot \mathrm{m}-\mathrm{DW} T S M)$ that is rated for $10 \mathrm{cN} \cdot \mathrm{m}$ to $1000 \mathrm{cN} \cdot \mathrm{m}$ has been completed and calibration service in this range has begun to the industry [7]. This development has accelerated the establishment of a calibration chain for HTDs.

One user in the medical field requested a digital HTD with the widest measuring range possible (for example, from 10 $\mathrm{cN} \cdot \mathrm{m}$ to $500 \mathrm{cN} \cdot \mathrm{m}$ ). The normative lower limit of an HTD is $20 \%$ of its nominal torque value according to ISO 6789: 2003 [6]. This paper also focuses on the problem where the HTD is used in the special range beyond the lower limit of $20 \%$ in conjunction with the limit of resolution in the reference device (TDT).

\section{EXPERIMENTAL CONDITIONS}

Experiments on the calibration chain were conducted as follows:

- Experiment 1: Calibration of the RTD using the TSM as the reference standard.

- Experiment 2: Calibration of the TDT using the RTD as the reference standard.

- Experiment 3: Testing verification of the HTD using the TDT as the standard.

These experimental conditions are described in the following subsections.

\subsection{Experiment 1}

A newly developed deadweight-type TSM with a rated capacity of $10 \mathrm{~N} \cdot \mathrm{m}$ was used (10-N·m-DWTSM) [7]. A picture of the machine is shown in Figure 2. The calibration range is from $10 \mathrm{cN} \cdot \mathrm{m}$ to $1000 \mathrm{cN} \cdot \mathrm{m}$. A torque transducer with a torque screwdriver shape (TP-6N-0707, manufactured by Showa Measuring Instruments Inc., cooperating with NMIJ) was

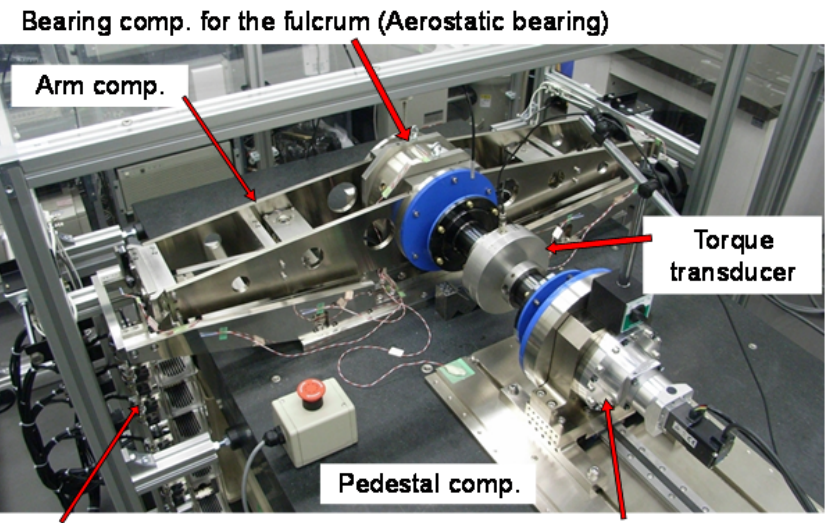

Weight loading comp.

Bearing comp. for the counter torque

Figure 2. Torque standard machine (10-N-m-DWTSM) for calibration of the reference torque screwdriver (RTD).

calibrated with a combination of an amplifier/indicator (MGCplus, amplifier type: ML38, manufactured by HBM $\mathrm{GmbH}$ ) as the RTD. The rated capacity is $600 \mathrm{cN} \cdot \mathrm{m}$, and the observed resolution of the RTD $r$ was $9.4 \mu \mathrm{N} \cdot \mathrm{m}$ (referring the definition of resolution in Section 3.1). A picture of the TP-6N0707 is shown in Figure 3.

Because of the limited calibration steps realized by the 10 $\mathrm{N} \cdot \mathrm{m}-\mathrm{DWTSM}$, calibration was performed in two ranges: from $10 \mathrm{cN} \cdot \mathrm{m}$ to $100 \mathrm{cN} \cdot \mathrm{m}$ and from $50 \mathrm{cN} \cdot \mathrm{m}$ to $600 \mathrm{cN} \cdot \mathrm{m}$. There were eight calibration steps, as follows:

. $10 \mathrm{cN} \cdot \mathrm{m}, 20 \mathrm{cN} \cdot \mathrm{m}, 30 \mathrm{cN} \cdot \mathrm{m}, 40 \mathrm{cN} \cdot \mathrm{m}, 50 \mathrm{cN} \cdot \mathrm{m}, 60 \mathrm{cN} \cdot \mathrm{m}$, $80 \mathrm{cN} \cdot \mathrm{m}$, and $100 \mathrm{cN} \cdot \mathrm{m}$,

· $50 \mathrm{cN} \cdot \mathrm{m}, 100 \mathrm{cN} \cdot \mathrm{m}, 150 \mathrm{cN} \cdot \mathrm{m}, 200 \mathrm{cN} \cdot \mathrm{m}, 300 \mathrm{cN} \cdot \mathrm{m}, 400$ $\mathrm{cN} \cdot \mathrm{m}, 500 \mathrm{cN} \cdot \mathrm{m}$, and $600 \mathrm{cN} \cdot \mathrm{m}$.

According to JMIF015 [8], which is the industrial guideline for the calibration method of TMDs issued by Japan Measuring Instruments Federation (JMIF), at first, two calibration cycles with increasing and decreasing loading steps were conducted after three instances of pre-loading up to the maximum torque at the $0^{\circ}$ mounting position. After changing the mounting position, one calibration cycle of increasing and decreasing loading steps was also conducted after one instance of preloading at the $120^{\circ}$ and $240^{\circ}$ mounting positions. The clockwise $(\mathrm{CW})$ and counterclockwise (CCW) torques were calibrated, respectively. Figure 4 shows the loading timetable for Experiment 1. The room temperature was $19.6{ }^{\circ} \mathrm{C}$ to $20.5^{\circ} \mathrm{C}$,

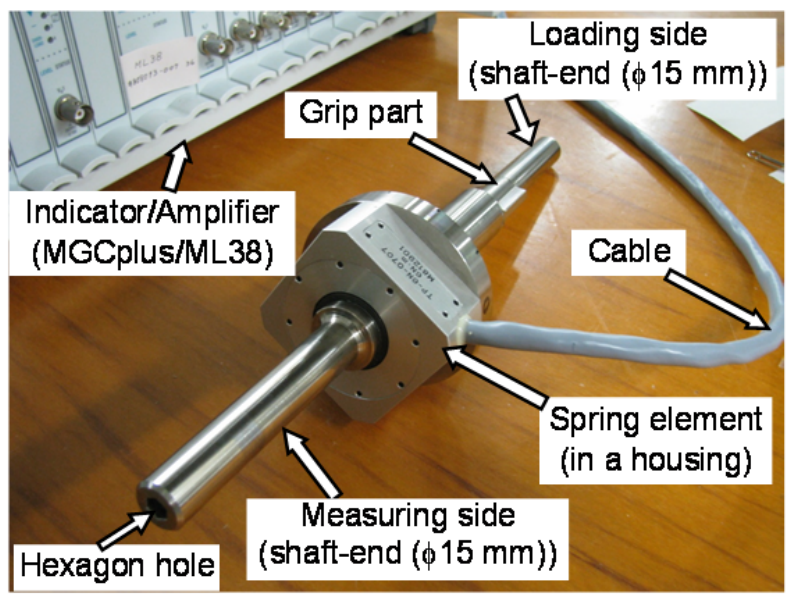

Figure 3. Torque transducer with torque screwdriver shape, which was manufactured by Showa Measuring Instrument, cooperating with NMIJ. 

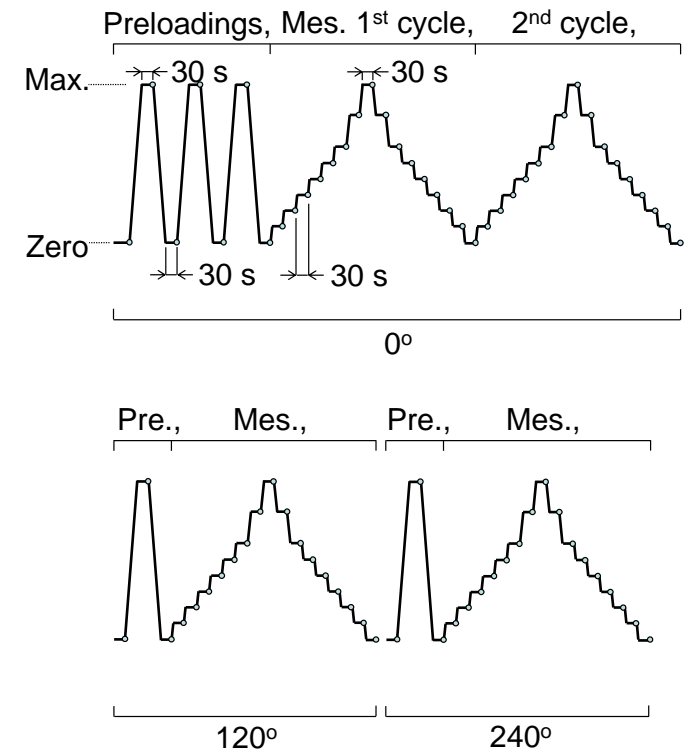

Figure 4. Loading timetable for the calibration of the reference torque screwdriver (RTD).

the relative humidity was $36 \%$ to $37 \%$ and the atmospheric pressure was $1013 \mathrm{hPa}$ to $1016 \mathrm{hPa}$ during the calibration.

\subsection{Experiment 2}

The RTD (TP-6N-0707 + MGCplus: ML38) was used as the reference standard. The calibration range was from $10 \mathrm{cN} \cdot \mathrm{m}$ to $600 \mathrm{cN} \cdot \mathrm{m}$. A TDT (TDT-600CN, manufactured by Tohnichi MFG Ltd.) was calibrated by installing the RTD on it (like a HTD). A picture of the TDT-600CN with the installed TP-6N0707 is shown in Figure 5. The calibration range specified by the manufacturer is from $20 \mathrm{cN} \cdot \mathrm{m}$ to $600 \mathrm{cN} \cdot \mathrm{m}$, but the authors investigated the calibration for one range from $10 \mathrm{cN} \cdot \mathrm{m}$ to $600 \mathrm{cN} \cdot \mathrm{m}$ in this experiment (the lower limit was extended). The resolution of the TDT $r$ was $0.2 \mathrm{cN} \cdot \mathrm{m}$. Eight calibration steps were conducted as follows:

- $10 \mathrm{cN} \cdot \mathrm{m}, 20 \mathrm{cN} \cdot \mathrm{m}, 50 \mathrm{cN} \cdot \mathrm{m}, 100 \mathrm{cN} \cdot \mathrm{m}, 200 \mathrm{cN} \cdot \mathrm{m}, 300$

$\mathrm{cN} \cdot \mathrm{m}, 400 \mathrm{cN} \cdot \mathrm{m}$, and $600 \mathrm{cN} \cdot \mathrm{m}$.

According to JMIF019 [9], which is another industrial guideline for the calibration method of torque wrench testers and torque testing machines issued by JMIF, two calibration cycles with increasing loading steps were conducted after three

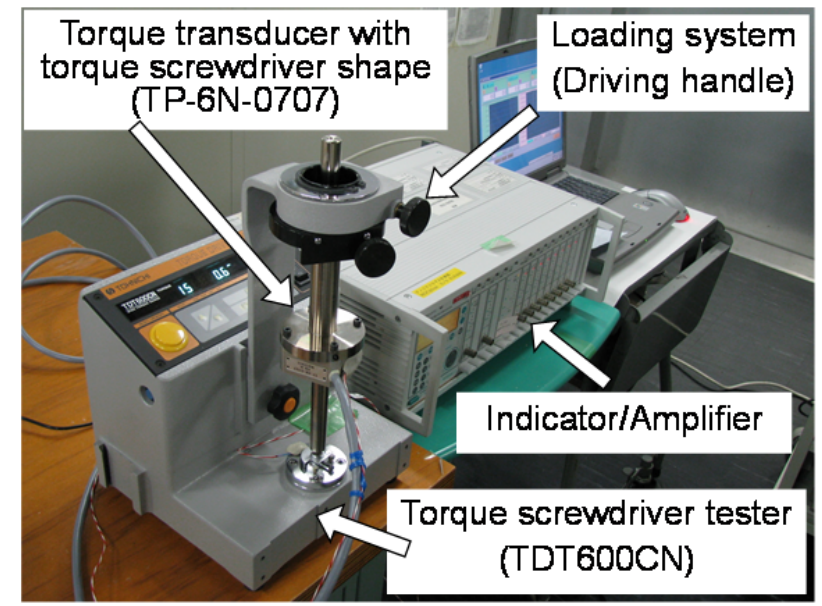

Figure 5. Torque screwdriver tester (TDT) with the reference torque screwdriver (RTD).
Preloadings, Mes. $1^{\text {st }}$ cycle, $2^{\text {nd }}$ cycle,
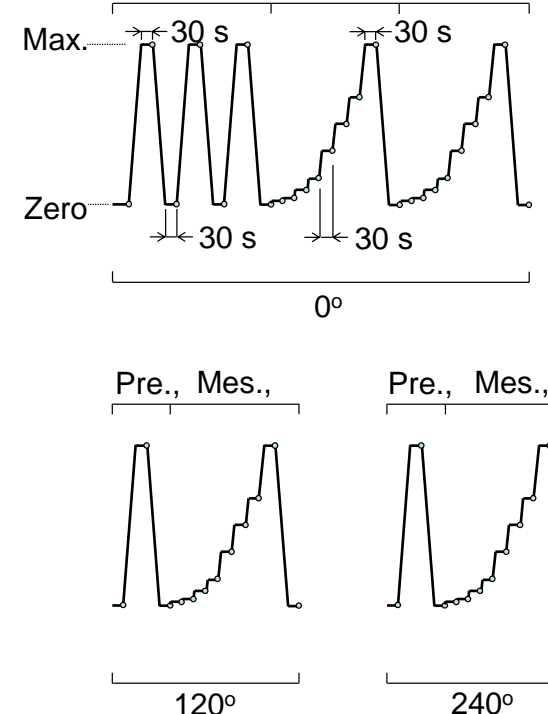

Pre., Mes.,

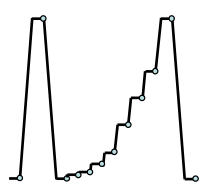

$240^{\circ}$

Figure 6. Loading timetable for the calibration of the Torque screwdriver tester (TDT).

instances of pre-loading up to the maximum torque at the $0^{\circ}$ mounting position. After changing the mounting position of the RTD, one calibration cycle of increasing steps was also conducted after one instance of pre-loading at the $120^{\circ}$ and $240^{\circ}$ mounting positions. $\mathrm{CW}$ and $\mathrm{CCW}$ torques were calibrated, respectively. Figure 6 shows the loading timetable for Experiment 2. The room temperature was $20.5{ }^{\circ} \mathrm{C}$ to 21.1 ${ }^{\circ} \mathrm{C}$, the relative humidity was $35 \%$ and the atmospheric pressure was $1001 \mathrm{hPa}$ to $1020 \mathrm{hPa}$ during the calibration.

\subsection{Experiment 3}

The TDT (TDT-600CN) was used as the reference standard. The calibrated range of the TDT was from $10 \mathrm{cN} \cdot \mathrm{m}$ to 600 $\mathrm{cN} \cdot \mathrm{m}$. The FTD-400CN (indicating type, the resolution: 1 $\mathrm{cN} \cdot \mathrm{m}$ ) and RTD-500CN (setting type, the smallest graduation: $5 \mathrm{cN} \cdot \mathrm{m}$ ) HTDs (both manufactured by Tohnichi MFG Ltd.) were tested by installing them on the TDT. Figure 7 shows pictures of the FTD-400CN and RTD-500CN installed on the TDT. The tested ranges were from $80 \mathrm{cN} \cdot \mathrm{m}$ to $400 \mathrm{cN} \cdot \mathrm{m}$ for the FTD $-400 \mathrm{CN}$ and from $100 \mathrm{cN} \cdot \mathrm{m}$ to $500 \mathrm{cN} \cdot \mathrm{m}$ for the RTD $500 \mathrm{CN}$.

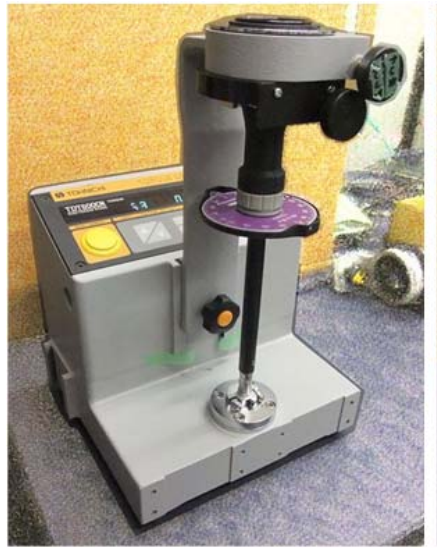

(a) Indicating type

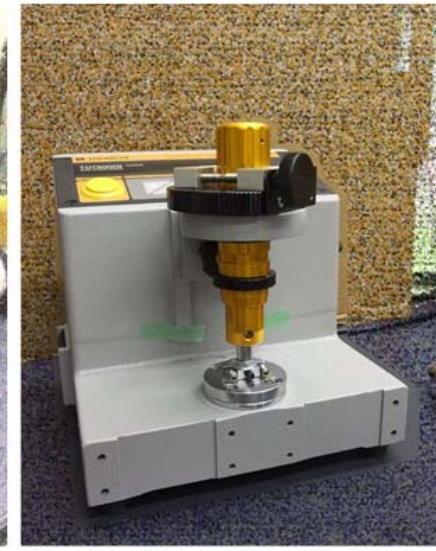

(b) Setting type

Figure 7. Hand torque screwdrivers (HTDs) installed on the torque screwdriver tester (TDT). 


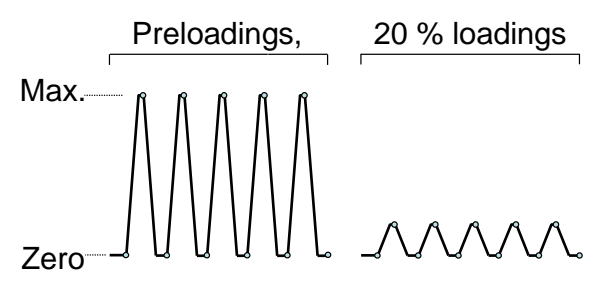

$60 \%$ loadings $\quad 100 \%$ loadings

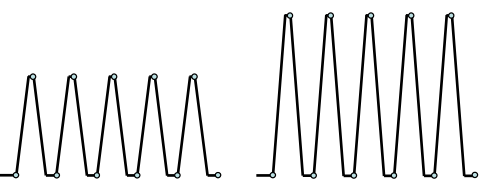

Figure 8. Loading timetable for the testing verification of Hand torque screwdrivers (HTDs).

According to ISO 6789 [6], three loading steps of $20 \%, 60$ $\%$, and $100 \%$ for maximum torque were conducted successively five times after pre-loading at the maximum torque five times. The CW and CCW torques were tested for the FTD $400 \mathrm{CN}$, and only the CW torque was tested for the RTD$500 \mathrm{CN}$. Figure 8 shows the loading timetable for Experiment 3. The room temperature was $20.3{ }^{\circ} \mathrm{C}$ to $20.5{ }^{\circ} \mathrm{C}$, the relative humidity was $33 \%$ to $34 \%$ and the atmospheric pressure was $1023 \mathrm{hPa}$ during the testing.

\section{RESULTS AND DISCUSSIONS}

\subsection{Experiment 1}

The uncertainty was evaluated according to JCG209S11 [10], which is a "guideline for the uncertainty evaluation of calibrations for torque meters and reference torque wrenches" issued by the International Accreditation Japan at the National Institute of Testing and Evaluation (IAJapan/NITE: the accreditation body for calibration and testing laboratories in Japan). The guideline was made mainly by the authors cooperating with Japanese industry, based on the research of calibration methods of TMDs [11][12]. Detailed explanation of the evaluation method is described as follows.

\section{Calibration results}

The measurement values at increasing torque $S$ ' and decreasing torque $S$ " are defined as the differences between the indicated values at the torque steps and the indicated value at the non-loading condition before starting the cycle. The calibration results $\overline{S^{\prime}}$ and $\overline{S^{\prime \prime}}$ are calculated according to Eqs. (1) as the means of the measurement values obtained from different mounting positions. The results measured during the second cycles are not included in the calculations of $\overline{S^{\prime}}$ and $\overline{S^{\prime \prime}}$ :

$$
\begin{aligned}
& \overline{S_{i}^{\prime}}=\frac{1}{n_{\text {rot }}} \sum_{\mathrm{e}=1}^{n_{\text {rot }}} S_{\mathrm{i} 1 \mathrm{e}}^{\prime} \\
& \overline{S^{\prime \prime}}=\frac{1}{n_{\text {rot }}} \sum_{\mathrm{e}=1}^{n_{\text {rot }}} S^{\prime \prime}{ }_{\mathrm{i} 1 \mathrm{e}}
\end{aligned}
$$

where $i, j(=1)$ and e are the indexes of calibration steps, cycles and different mounting position series, and $n_{\text {rot }}$ indicates the total number of mounting positions.

\section{Reproducibility with different mounting positions}

The relative reproducibility with different mounting positions $b_{\mathrm{i}}$ is calculated according to Eq. (2) as the experimental standard deviation of the measurement values during the first cycle of each mounting position:

$$
b_{\mathrm{i}}=\frac{1}{\left|\overline{S_{\mathrm{i}}^{\prime}}\right|} \sqrt{\frac{1}{\left(n_{\mathrm{rot}}-1\right)} \sum_{\mathrm{e}=1}^{n_{\mathrm{rot}}}\left(S_{\mathrm{i} 1 \mathrm{e}}^{\prime}-\overline{S_{\mathrm{i}}^{\prime}}\right)^{2}}
$$

The relative standard uncertainty $w_{\text {rot_rtd,i }}$ is calculated according to Eq. (3) as the experimental standard deviation of the mean:

$$
w_{\text {rot_rtd, } \mathrm{i}}{ }^{2}=\frac{1}{n_{\text {rot }}} b_{\mathrm{i}}^{2}
$$

The results measured during the second cycles are not included in the calculation of $b_{1}$.

\section{Repeatability with an unchanged mounting position}

The relative repeatability with an unchanged mounting position $b_{\mathrm{i}}^{\prime}$ is calculated according to Eq. (4) when the cycle is repeated twice for only $0^{\circ}$ series:

$$
b_{\mathrm{i}}^{\prime}=\frac{S_{\mathrm{i} 21}^{\prime}-S_{\mathrm{i} 11}^{\prime}}{\left|\frac{1}{n_{\text {rep }}} \sum_{\mathrm{j}=1}^{n_{\text {rep }}} S_{\mathrm{ij} 1}^{\prime}\right|}
$$

where the $n_{\text {rep }}$ is the number of cycles for the same series, and $S_{\mathrm{ij} 1}^{\prime}$ indicates the measurement value at step $i$, cycle $\mathrm{j}$ and for the first series $(e=1)$.

The relative standard uncertainty $w_{\text {rep_rtd,i }}$ is calculated according to Eq. (5), by determining $b_{\mathrm{i}}^{\prime}$ as a half-width of the rectangular distribution:

$$
w_{\text {rep_rtd,i }}{ }^{2}=\frac{1}{3} b_{\mathrm{i}}^{\prime 2}
$$

\section{Deviation due to interpolation}

An interpolation equation for the increasing torque is determined via the least squares method using the calibration results $\overline{S^{\prime}{ }_{i}}(i=1$ to $n)$. The interpolation equation relates the measurement value $S$ ' and the torque $T$ as follows:

$$
S^{\prime}=A_{0}+A_{1} \cdot T+A_{2} \cdot T^{2}+\cdots+A_{\mathrm{m}} \cdot T^{\mathrm{m}}
$$

Whether or not the interpolation equation includes a constant term depends on the manual of the calibration laboratory or the specifications from the client although the constant term was included in this experiment.

If so be required, the interpolation equation for the decreasing torque may be determined as follows:

$$
S^{\prime \prime}=A_{0}{ }^{\prime}+A_{1}{ }^{\prime} \cdot T+A_{2}{ }^{\prime} \cdot T^{2}+\cdots+A_{\mathrm{m}}{ }^{\prime} \cdot T^{\mathrm{m}}
$$

The interpolation equation of the decreasing torque, Eq. (6b), must be determined as the calculated values at the maximum torque $T_{\max }$ coincides with the value calculated by the interpolation equation of increasing torque, Eq. (6a). The authors also calculated the interpolation equation of the decreasing torque in this experiment. However, the RTD was not subsequently used for the calibration of the TDT in decreasing torque steps.

The relative deviation due to the interpolation $f_{a, i}$ is calculated according to Eq. (7) as the difference between the calculated 
value obtained by the interpolation equation $S_{a, \mathrm{i}}^{\prime}$ and the calibration result $\overline{S^{\prime}}$ :

$$
f_{\mathrm{a}, \mathrm{i}}=\frac{\overline{S_{\mathrm{i}}^{\prime}}-S_{\mathrm{a}, \mathrm{i}}^{\prime}}{\left|S_{\mathrm{a}, \mathrm{i}}^{\prime}\right|}
$$

The relative standard uncertainty associated with interpolation $w_{\text {int_rtd,i }}$ is calculated according to Eq. (8), by determining $f_{a, i}$ as a half-width of the rectangular distribution:

$$
w_{\text {int_rtd,i }}{ }^{2}=\frac{1}{3} f_{\mathrm{a}, \mathrm{i}}{ }^{2}
$$

The degree $\left(1^{\text {st }}, 2^{\text {nd }}\right.$ or $\left.3^{\text {rd }}\right)$ of the interpolation equation to be used is recommended to meet the following condition:

$$
\begin{aligned}
& n<5 ; 1^{\text {st }} \text { degree, } \\
& n<8 ; 2^{\text {nd }} \text { degree, } \\
& n \geq 8 ; 3^{\text {rd }} \text { degree. }
\end{aligned}
$$

Then, the interpolation of $3^{\text {rd }}$ degree was used for this experiment.

If the RTD directly indicates values in the unit of torque and the measurement values cannot be electronically fitted to the interpolation curve of the calibration result, then rather than $w_{\text {int_rtd, }}$ the relative standard uncertainty of the deviation due to indication $w_{\text {ind_rtd }}$ should be used. A detailed explanation, however, is not presented herein.

\section{Zero error}

The relative zero error $f_{0, \mathrm{e}}$ is calculated according to Eq. (9) as the absolute value of deviation between the indicated values at the non-loading conditions before starting and after finishing the cycle. The calculation of $f_{0, \mathrm{e}}$ does not include values obtained in calibration cycles with only increasing torque:

$$
f_{0, \mathrm{e}}=\frac{S_{01 \mathrm{e}}^{\prime \prime}-S_{01 \mathrm{e}}^{\prime}}{\left|S_{\mathrm{n} 1 \mathrm{e}}^{\prime}\right|}
$$

where $S_{01 \mathrm{e}}^{\prime}$ and $S_{01 \mathrm{e}}^{\prime}$ are the zero values indicated before starting and after finishing the cycle for the first cycle and series e. $S_{\text {n1e }}^{\prime}$ shows the measurement value at the maximum torque in series e.

The relative standard uncertainty due to zero drift $w_{\text {zer_rtd,i }}$ is calculated according to Eq. (10), by determining the maximum value of $f_{0, \mathrm{e}}$ as a half-width of the rectangular distribution:

$$
w_{\text {zer_trd }}{ }^{2}=\frac{1}{3} f_{0, \max }^{2}
$$

\section{Hysteresis}

The relative reversibility error (hysteresis) $b_{\mathrm{i}}$ is calculated according to Eq. (12) as the mean of the absolute differences between measurement values at increasing and decreasing torque steps in the same cycle:

$$
b_{\mathrm{i}}=\frac{\frac{1}{n_{\text {rot }}} \sum_{\mathrm{e}=1}^{n_{\text {rot }}}\left|S_{\mathrm{i} 1 \mathrm{e}}^{\prime \prime}-S_{\mathrm{i} 1 \mathrm{e}}^{\prime}\right|}{\left|\overline{S_{\mathrm{i}}^{\prime}}\right|}
$$

where $S$ ”ie $_{\text {ie }}$ is the measurement value of the decreasing torque at step $\mathrm{i}$ at the first cycle for series e. The values measured in the cycle including only increasing torque are not included in the calculation of $h_{\mathrm{i}}$.

The relative standard uncertainty due to reversibility $w_{\text {rev_rtd,i }}$ is calculated according to Eq. (12), by determining $h_{\mathrm{i}}$ as a halfwidth of the rectangular distribution:

$$
w_{\text {rev_rtd,i }}{ }^{2}=\frac{1}{3} h_{\mathrm{i}}{ }^{2}
$$

The relative reversibility error, however, was not considered in this experiment as mentioned later.

\section{Resolution}

The resolution $r$ of the indicator is defined as the smallest fraction of a scale division that is readable in the case of analogue scale. In the case of digital scale, $r$ is defined to be one increment of the last active number of the numerical indicator, provided that the indication does not fluctuate when the RTD is under the non-loading condition. If the indication fluctuates under the non-loading condition by more than one increment, the resolution is equal to a half-width of the fluctuation (here, the fluctuation is two counts when the last active number is fluctuated from 0 to 1 , and three counts when it is fluctuated from 0 to 2 , for example). $r$ must be converted to and stated in units of torque as expressed in Section 2.1.

The relative standard uncertainty due to resolution $w_{\text {res_rtd,i }}$ is calculated according to Eqs. (13), by determining $r$ as a halfwidth or a whole-width of the rectangular distribution depending on the fluctuation:

$$
\begin{aligned}
& w_{\text {res_rtd,i }}{ }^{2}=\frac{2}{3}\left(\frac{r}{2 T_{\mathrm{i}}}\right)^{2} \text { (non-fluctuation) } \\
& w_{\text {res_rtd,i }}{ }^{2}=\frac{2}{3}\left(\frac{r}{T_{\mathrm{i}}}\right)^{2} \text { (fluctuation) }
\end{aligned}
$$

where $T_{\mathrm{i}}$ is the torque at each torque step. $w_{\text {res_rtd,i }}$ is multiplied by $\sqrt{2}$ considering that the measurement value is obtained as the difference between the indicated values of the loaded torque step and non-loading zero step before starting the cycle (double readings).

\section{Uncertainty of the calibration result}

The relative expanded uncertainty $W_{\text {rtd_cal }}$ of calibration for the RTD is calculated from the relative combined standard uncertainty $w_{\text {tsm }}$ of torque realized by the TSM and the relative combined standard uncertainty $w_{c_{-} \text {rtd }}$ ascribable to the measurement of the RTD, according to the following equation:

$$
W_{\text {rtd_cal,i }}=k \cdot w_{c_{-} \text {rtd_cal,i }}=k \cdot \sqrt{w_{\text {tsm }}^{2}+w_{\text {c_rtd }}^{2}}
$$

where the coverage factor $k$ is equal to two and its interval was estimated to have a level of confidence of approximately $95 \%$. The authors also evaluated the effective degree of freedom and confirmed sufficient large number. $w_{\text {tsm }}$ has been evaluated as $3.3 \times 10^{-5}[7] . w_{\text {c_rtd }}$ is calculated as:

$$
w_{\text {c_rtd,i }}=\frac{\sqrt{w_{\text {rot_rtd,i }}{ }^{2}+w_{\text {rep_rtd,i }}^{2}+w_{\text {int_rtd,i }}^{2}}}{+w_{\text {zer_rtd,i }}^{2}+w_{\text {rev_rtd,i }}^{2}+w_{\text {res_rtd,i }}^{2}}
$$

In the case of the evaluation of only increasing torque, $w_{c_{\_} \text {rtd }}$ is calculated from:

$$
\begin{aligned}
& w_{\text {c_trd,i }}=\sqrt{w_{\text {rot_rtd,i }}^{2}+w_{\text {rep_rtd,i }}^{2}+w_{\text {int_rtd, } i}^{2}} \\
& \overline{+w_{\text {zer_rtd,i }}^{2}+w_{\text {res_rtd,i }}^{2}}
\end{aligned}
$$

Eq. (15b) was used for the calculation in this experiment.

The results of the uncertainty evaluation are shown in Figure 9. The maximum relative expanded uncertainty for each calibration range was as follows:
$10 \mathrm{cN} \cdot \mathrm{m}-100 \mathrm{cN} \cdot \mathrm{m}$ :
CW $0.024 \%$, CCW $0.031 \%$,
$50 \mathrm{cN} \cdot \mathrm{m}-600 \mathrm{cN} \cdot \mathrm{m}: \quad \mathrm{CW} 0.021 \%$, CCW $0.020 \%$. 


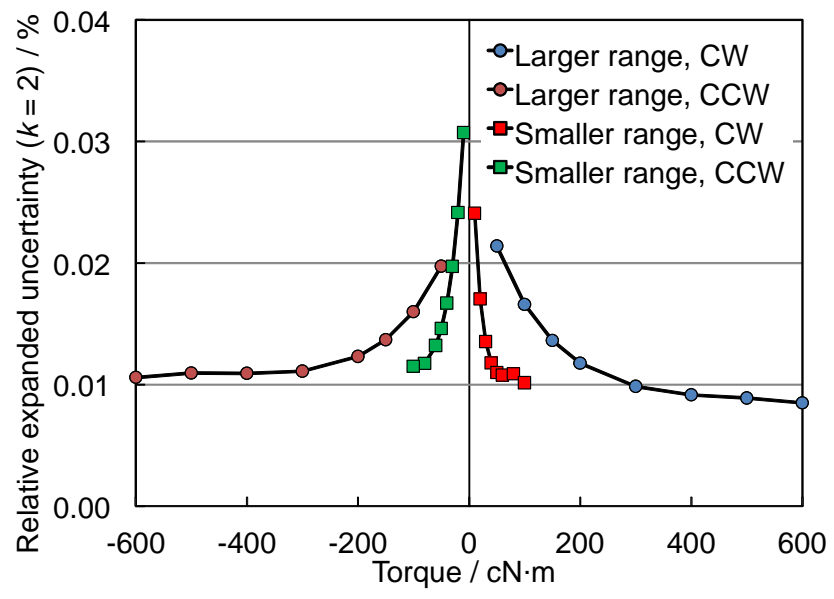

Figure 9. Uncertainty evaluation results of the calibration for the RTD

Sufficient small uncertainties could be obtained because the RTD was directly calibrated by using the TSM. The relative uncertainty must be larger than the level of $0.02 \%$ when the RTD is calibrated by using the TCM of a calibration laboratory as shown in Figure 1.

The interpolation equations obtained in the experiment 1 were used for the successive calibration of the TDT described in Section 2.2.

\subsection{Experiment 2}

The uncertainty was evaluated according to JCG209S21 [13], which is an another guideline for the uncertainty evaluation of calibrations for torque wrench testers and torque testing machines issued by IAJapan/NITE. The guideline was also made mainly by the authors cooperating with Japanese industry. Detailed explanation of the evaluation method is described (but only increasing torque) as follows.

\section{Uncertainty of the calibration result}

First, the relative expanded uncertainty of the calibration is expressed by the following equation:

$$
W_{\text {cal_tdt,i }}=k \cdot \frac{\sqrt{w_{\text {rot_tdt,i }}^{2}+w_{\text {rep_tdt,i }}^{2}+w_{\text {int_tdt,i }}^{2}}}{+w_{\text {zer_tdt,i }}^{2}+w_{\text {res_tdt,i }}^{2}+w_{\text {rtd }}^{2}}
$$

Calibration results $\overline{S^{\prime}}$, the uncertainty contribution for the reproducibility with the change in the mounting position $w_{\text {rot_tdt }}$, that for the repeatability without the change in the mounting position $w_{\text {rep_tdt, }}$ that due to the interpolation $w_{\text {int_tdt, }}$ that due to the resolution $w_{\text {res_tdt }}$ and the the coverage factor $k$ are evaluated in the same way as described in Section 3.1.

\section{Zero error}

The loading timetable was only increasing torque in this calibration. But the relative zero error $f_{0, \mathrm{e}}$ and the relative standard uncertainty $w_{\text {zer_tdt }}$ is calculated according to Eq. (9) and (10) as well. In this case, the uncertainty tends to be larger than the zero error for the cycle of increasing and decreasing torque because unloading from the maximum torque suddenly occurs in the only increasing torque cycle. This would be controversy in the future. However, fortunately, the TDT showed null values of the zero error in all cycles in this experiment because of low resolution in the TDT.

\section{Uncertainty of $R T D$ as a reference standard}

The relative combined uncertainty $w_{\text {rtd }}$ when the RTD is used as a reference standard is calculated from the relative combined standard uncertainty $w_{c_{-} \text {rtd_cal }}$ calculated by Eq. (14), the relative standard uncertainty due to temperature variation $w_{\text {rtd_tmp }}$, and the relative standard uncertainty of long term stability of the RTD $w_{\text {rtd_lgstb }}$, according to the following equation:

$$
w_{\text {rtd }}=\sqrt{w_{\text {c_rtd_cal }}^{2}+w_{\text {rtd_tmp }}^{2}+w_{\text {rtd_lgstb }}^{2}}
$$

$w_{\text {rtd_tmp }}$ is calculated by the following equation:

$$
w_{\text {rtd_tmp }}{ }^{2}=\frac{\beta^{2}}{3}\left(\frac{\Delta t_{\text {meas }}}{2}\right)^{2}
$$

where $\beta$ is temperature coefficient of output sensitivity of the RTD and $\Delta t_{\text {meas }}$ is temperature variation. The temperature correction of output value of RTD is available if necessary. This term was small in this experiment because the temperature coefficient is $-1.4 \times 10^{-4} \mathrm{~K}^{-1}$ and the temperature variation was $1.5 \mathrm{~K}$ at the maximum. $w_{\text {rtd_tmp }}$ was $6.1 \times 10^{-5}$.

$w_{\text {rtd_lgstb }}$ is calculated by the following equation:

$$
w_{\text {rtd_lgstb }}=\frac{1}{\left|\overline{S_{\text {c_mean }}}\right|} \sqrt{\left.\frac{1}{\left(n_{\text {cal }}-1\right)} \sum_{\mathrm{c}=1}^{n_{\text {cal }}} \overline{\left(S_{\mathrm{c}}\right.}-\overline{S_{\mathrm{c} \_ \text {mean }}}\right)^{2}} \text {, }
$$

provided the RTD has been calibrated more than three times $\left(n_{\text {cal }} \geq 3\right)$ in the pre-determined calibration period (generally 26 months). Here, $\overline{S_{c}}$ is a calibration result of each time and $\overline{S_{\text {c_mean }}}$ the mean value of calibration results for all time. It would be acceptable empirically determine $w_{\text {rtd_lgstb }}$ if the calibration time is less than three. The authors used empirically $0.02 \%$ for $w_{\text {rtd_lgstb. }}$.

The result of the uncertainty evaluation of calibration for the TDT is shown in Figure 10. The maximum relative expanded uncertainties for the various lower limits of the calibration were as follows:

$10 \mathrm{cN} \cdot \mathrm{m}-600 \mathrm{cN} \cdot \mathrm{m}: \quad \mathrm{CW} 6.6 \%$, CCW $2.9 \%$,

$20 \mathrm{cN} \cdot \mathrm{m}-600 \mathrm{cN} \cdot \mathrm{m}: \quad \mathrm{CW} 2.6 \%$, CCW $2.0 \%$,

$50 \mathrm{cN} \cdot \mathrm{m}-600 \mathrm{cN} \cdot \mathrm{m}: \quad \mathrm{CW} 1.1 \%$, CCW $0.62 \%$,

$100 \mathrm{cN} \cdot \mathrm{m}-600 \mathrm{cN} \cdot \mathrm{m}:$ CW $0.75 \%$, CCW $0.24 \%$.

In this evaluation, the uncertainty contribution of the interpolation ( $w_{\text {int_tdt }}$ ) was taken into consideration instead of the indication error ( $w_{\text {ind_tdt }}$ ); i.e., the authors converted the indicated values into the reference values by using the interpolation equations when the TDT was used for the testing of the HTDs described in Section 2.3 although the TDT has a digital indication display (by the direct torque unit expression).

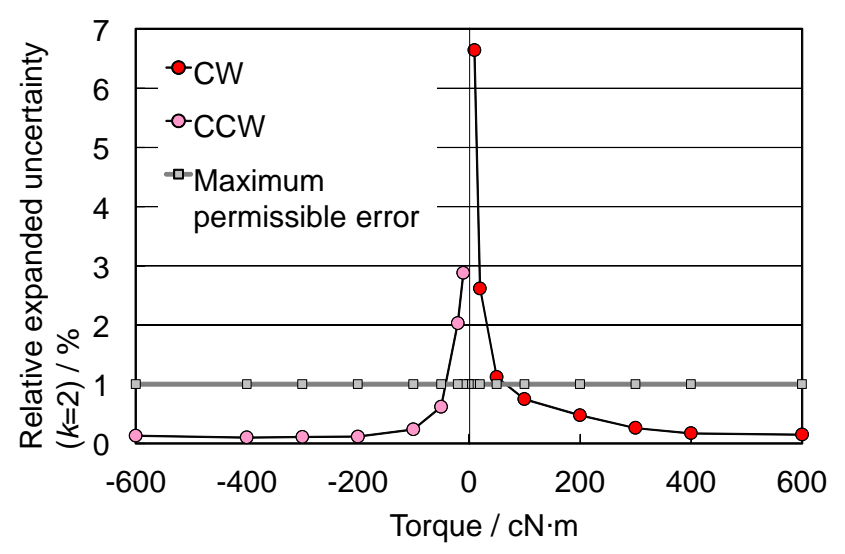

Figure 10. Uncertainty evaluation results of the calibration for the TDT.. 


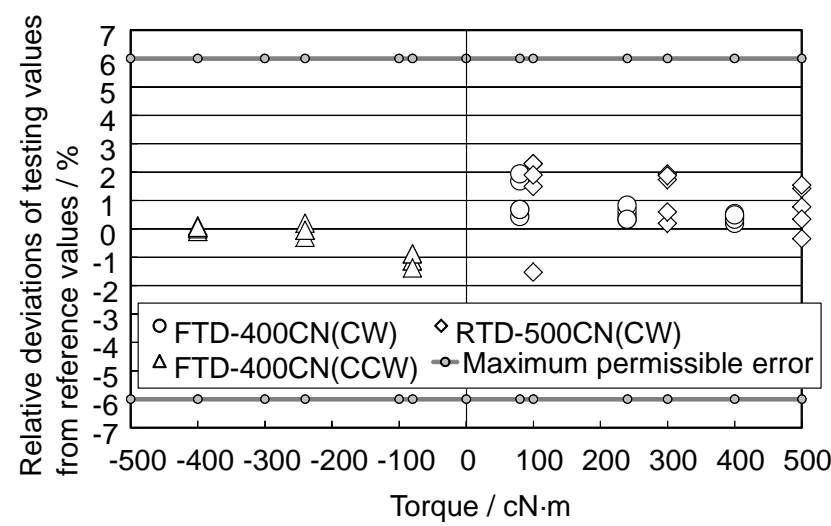

Figure 11. Testing results for HTDs.

It was found that the uncertainty exceeded $1 \%$, even if the interpolation equations were used when including a lower limit of 10,20 , or $50 \mathrm{cN} \cdot \mathrm{m}$. Both the users and manufacturers of TDTs should pay attention to the choice of the lower limit and consider the uncertainty. In addition, the authors insist that the normative description of "the maximum permissible uncertainty of the torque tool calibration devices shall be less than $1 \%$." in ISO 6789 should be clarified; i.e., the method of uncertainty evaluation for the TDT calibration should be defined and harmonized.

\subsection{Experiment 3}

Figure 11 shows test results for two different HTDs. In all of the five successive measurements at each step, the relative deviations from the reference standard (TDT) were within $6 \%$ of the maximum permissible deviation prescribed in ISO 6789 for both the FTD-400CN and RTD-500CN. These HTDs were recognized to conform with ISO 6789 because the maximum uncertainty of the TDT was within $1 \%$ in the tested ranges from $80 \mathrm{cN} \cdot \mathrm{m}$ to $500 \mathrm{cN} \cdot \mathrm{m}$. However, if the TDT is used, including the lower range from $10 \mathrm{cN} \cdot \mathrm{m}$ to $50 \mathrm{cN} \cdot \mathrm{m}$ as described in Section 1, the testing results must be out of conformity with ISO 6789.

The uncertainty was not evaluated for this test results. After discussion with manufacturers and users of HTDs in Japan, it was found that the uncertainty of measurement for the results of testing HTDs is not yet required. On the other hand, the challenge for calculation of the uncertainty has started [4]. The authors will continue to discuss the problem with related organizations.

\section{CONCLUSIONS}

The authors experimentally realized a complete calibration chain from the national torque standard to the hand torque screwdriver (HTD), where the appropriate calibration methods and uncertainty evaluations were applied.

When torque screwdriver testers (TDTs) are used as reference standards for the testing of HTDs, testing organizations should pay attention to the fact that the calibration uncertainties of the TDTs must be within the maximum permissible uncertainty prescribed in ISO 6789 for the entire testing range of the HTDs.

Here, as shown in Figure 1, it would be acceptable even if the first-grade accredited laboratories will calibrate RTDs with an uncertainty of approximately $0.2 \%$ for calibrators or users of TDTs instead of the values presented in Section 3.1 (approx. $0.02 \%-0.03 \%$ ). The authors, therefore, insist that it would be sufficiently possible for the Japanese industry to establish the hierarchy proposed in Figure 1.

\section{REFERENCES}

[1] P. D. Hohmann, "Advantages of Traceability by using torque transfer standard TTS," Proc. of XIII IMEKO World Congress, Sept. 1994, Torino, Italy, pp. 253-256.

[2] A. Brüge, D. Röske, D. Mauersberger and K. Adolf, "Influence of Cross Forces and Bending Moments on Reference Torque Sensors for Torque Wrench Calibration," Proc. XIX IMEKO World Congress, Sept. 2009, Lisbon, Portugal, pp.356-361.

[3] K. Ogushi, A. Nishino, K. Maeda and K. Ueda, "Calibration of a torque wrench tester using a reference torque wrench," SICE Annual Conference 2011, Sept, 2011, Tokyo, Japan, pp. 411-416.

[4] D. Röske, "ISO 6789:2003 Calibration Results of Hand Torque Tools with Measurement Uncertainty - Some Proposals," Proc. XX IMEKO World Congress, Sept, 2012, Busan, Republic of Korea,USB flash drive.

[5] K. Ogushi, A. Nishino, K. Maeda and K. Ueda, "Advantages of the calibration chain for hand torque screwdrivers traceable to the national torque standard," SICE Annual Conference 2012, Akita, Japan, Sept. 2012, USB flash drive.

[6] JIS B 4652 (ISO 6789: 2003), "Hand torque tools - Requirements and test methods," Japanese Standards Association, 2008 (in Japanese).

[7] A. Nishino, K. Ogushi and K. Ueda, "Uncertainty evaluation of a $10 \mathrm{~N} \cdot \mathrm{m}$ dead weight torque standard machine and comparison with an $1 \mathrm{kN} \cdot \mathrm{m}$ dead weight torque standard machine," Measurement, 49 (2014), pp. 77-90.

[8] JMIF015, "Guideline for calibration laboratories of torque measuring devices", Japan Measuring Instruments Federation, 2004 (in Japanese).

[9] JMIF019, "Guideline for Calibration Laboratories of Torque Testing Machines and/or Torque Wrench Tester," Japan Measuring Instruments Federation, 2007 (in Japanese).

[10] JCG209S11-05, "JCSS Guideline on Uncertainty Estimation Torque Meters and Reference Torque Wrenches," International Accreditation Japan, National Institute of Testing and Evaluation, 2012 (in Japanese).

[11] K. Ohgushi, T. Ota and K. Ueda, "Experimental Study on the Uncertainty in Static Calibration of a Torque Measuring Device 1st Report: Verification of Repeatability, Reproducibility and Zero Error-," 'Trans of the SICE, 39-7 (2003), pp. 624-630 (in Japanese).

[12] K. Ohgushi, T. Ota and K. Ueda, "Experimental Study on the Uncertainty in Static Calibration of a Torque Measuring Device 2nd Report: Verification of Interpolation Error, Reversibility and Resolution- (in Japanese)," Trans of the SICE, 39-7 (2003), pp. 631-636 (in Japanese).

[13] JCG209S21-02, "JCSS Guideline on Uncertainty Estimation Torque Wrench Testers and Torque Testing Machines," International Accreditation Japan, National Institute of Testing and Evaluation, 2012 (in Japanese). 